\title{
Doping-induced modulation of electronic, optical and wetting properties of $\mathrm{CeO}_{2}$
}

\author{
Authors: D. Mamedov ${ }^{1,2^{*}}$ and S. Zh. Karazhanov ${ }^{1,2}$ \\ ${ }^{1}$ Department for Solar Energy, Institute for Energy Technology, 2027 Kjeller, Norway \\ ${ }^{2}$ Department of Materials Science, National Research Nuclear University, 115409 Moscow, Russia
}

\begin{abstract}
Recently, $\mathrm{CeO}_{2}$ as well as other Rare-Earth Oxides (REOs) have become known as water repelling materials which enables their usage in glass industry. In this study, we investigate electronic, optical and wetting properties of pure and doped $\mathrm{CeO}_{2}$ by first-principles calculations. It is established that introduction of tetravalent doping atoms $\left(\mathrm{Zr}, \mathrm{Ti}, \mathrm{Sn}\right.$ and $\mathrm{Si}$ ) significantly modifies the optical response of $\mathrm{CeO}_{2}$ in the visible range by shifting the absorption edge which also effects on the refractive index of the material. For these systems, the water contact angle has been computed through adsorption energy of water layers. We report the intrinsic hydrophilicity of the low-index surfaces of $\mathrm{CeO}_{2}$, which is enhanced by introduction of the impurity atom. Influence of the dopants on the oxygen vacancy formation energy $E_{f}\left(V_{O}\right)$ is considered and discussed with respect to its possible effect on the hydrophobic behavior of $\mathrm{CeO}_{2}$. It is found out that all the considered doping atoms reduce $E_{f}\left(V_{O}\right)$, resulting in enhanced adsorption of the air hydrocarbons at the surface, which leads to an increased water contact angle. Based on the obtained results, an assessment on the applicability of doped $\mathrm{CeO}_{2}$ in glass industry is made. It is concluded that $\mathrm{Zr}$-doped $\mathrm{CeO}_{2}$ possesses the most prominent properties among considered systems for the application as transparent layer.
\end{abstract}

\section{Keywords}

Doped cerium dioxide; Contact angle; Density functional theory; Defect chemistry; Optical properties.

\footnotetext{
* Corresponding author: damir.mamedov@ife.no
} 


\section{Introduction}

Cerium dioxide $\mathrm{CeO}_{2}$ (ceria) is a rare-earth metal oxide which found its application in different technological areas as catalytic ${ }^{1-3}$, ionic conductive ${ }^{4-6}$, and antimicrobial material ${ }^{7,8}$. Possessing fluorite crystal structure, $\mathrm{CeO}_{2}$ has a unique electronic configuration which allows its properties to be modified in a wide range by doping and $\mathrm{Ce}^{+4}$ reduction ${ }^{9,10}$. It was shown previously that another application of $\mathrm{CeO}_{2}$ can be to use it as a hydrophobic material ${ }^{11}$. Due to its wetting behavior together with transparency to visible light, cerium dioxide is a fine candidate for robust inorganic hydrophobic coatings which can be applied for example in windows for cars and buildings ${ }^{12,13}$. In addition, $\mathrm{CeO}_{2}$ is potentially capable to be used as anti-soiling and self-cleaning coatings for decreasing maintenance costs for PV-systems ${ }^{14,15}$, since a superhydrophobic condition with contact angle above $150^{\circ}$ can be achieved. The reasons for the hydrophobic properties for $\mathrm{CeO}_{2}$ still remain unclear. The original explanation was grounded on shield effect of cerium outer electrons, protecting $\mathrm{Ce}-4 f$ valence states from interaction with water oxygen atoms $\mathrm{s}^{11,16}$. The following studies showed controversial results, confirming dependence ${ }^{17}$ and independence ${ }^{18}$ of the wetting properties of magnetron sputtered $\mathrm{CeO}_{2}$ on $\mathrm{O} / \mathrm{Ce}$ ratio at surface. It was later found that the as-prepared $\mathrm{CeO}_{2}$ showed a low contact angle that increased over time in atmosphere or low-vacuum systems ${ }^{19}$. This evolution of wetting properties was later attributed to adsorption of hydrocarbon molecules from the environment on the surface of $\mathrm{CeO}_{2}$. Moreover, the initial hydrophilicity of $\mathrm{CeO}_{2}$ can be restored by removal of the adsorbed species from the material surface, which has been confirmed in a number of different works ${ }^{20-22}$.

One of the practical limitations of application of $\mathrm{CeO}_{2}$ in the glass industry is its high refractive index, which varies between 2.25 and 3.3 in the visible light range ${ }^{23-25}$,resulting in high reflection of light from the surface of the glass. The reflectivity of $\mathrm{CeO}_{2}$ can be tuned by doping which results in reduced refractive index and increased band gap. Impurities such as $\mathrm{Zr}^{26-29}, \mathrm{Ti}^{28}, \mathrm{Y}^{30}, \mathrm{Sn}^{31}, \mathrm{Si}^{32}, \mathrm{Mn}^{33}$ and various lanthanides ${ }^{34,35}$ all have the potential to tune the optical parameters of $\mathrm{CeO}_{2}$.

Theoretical considerations performed for doped systems have mostly focused on their application in catalytic or solid oxide fuel cells (SOFC) technologies and barely covered their effect on hydrophobicity. In a recent paper ${ }^{36}$, the influence of doping atoms on the water contact angle has been attributed to the change of atom-to-atom distance on the $\mathrm{CeO}_{2}$ surface, which defines the energetic outcome for the water molecules to be adsorbed ${ }^{37}$. However, such approach does not cover the effect of the electronic configuration near the surface on the wetting properties. Moreover, such doping influences the chemistry of oxygen vacancies, including their concentration at the surface. The intrinsic defects can be used by surrounding hydrocarbon molecules for the better attraction to the surface ${ }^{38}$ which enhances hydrophobicity.

Study of water contact angle for $\mathrm{CeO}_{2}$ has been addressed by first principles calculations for different configurations of the water molecule, showing high contact angle for ideal low-index surfaces in agreement with the initial idea of intrinsic hydrophobicity ${ }^{36,39}$, but contradicting the above-mentioned experimental results. The reason for the discrepancy between the theoretical and experimental results is not clear and might be explained by surface imperfections, such as attached hydroxyl groups. They cause rearrangement of the neighboring atoms of $\mathrm{CeO} 2$, which affects the electronic structure and modifies the wetting behavior of the surface. Additionally, the hydroxyl groups on the surface could serve as pinpoints for airborne hydrocarbons to attach ${ }^{38}$ which are responsible for gradual change of $\mathrm{CeO}_{2}$ hydrophobicity. However, the considered models imply a relatively high density of such defects, which is difficult to achieve in experiment with precise control of grains orientation. For instance, using atomic layer deposition (ALD) for synthesis of $\mathrm{CeO}_{2}$ thin films results in water contact angles of not more than $53^{\circ}$ regardless of the crystallographic indexes of the $\mathrm{CeO}_{2}$ surfaces investigated ${ }^{40}$.

The current work aims to study electronic structure and optical properties of ideal and doped $\mathrm{CeO}_{2}$ by first principles calculations. Range separated parameter $a$ has been estimated and the study of electronic and optical properties have been performed by using the calculated $a$ as an input to the hybrid functional. The influence of the introduction of tetravalent doping atoms $(\mathrm{Zr}, \mathrm{Ti}, \mathrm{Sn}$ and $\mathrm{Si}$ ) on the electronic structure, optics, oxygen vacancy formation energies and hydrophobicity of $\mathrm{CeO}_{2}$ has been investigated. For these systems, the water contact angle has been computed through adsorption energy of water layers. The applicability of different dopants for $\mathrm{CeO}_{2}$ in glass industry is evaluated based on the hydrophobic behavior of the systems as well as their optical response to visible light. 


\section{Computational details}

\subsection{Structural and optical properties}

Calculations were performed using Density Functional Theory (DFT) implemented in Vienna Ab Initio Simulation Package (VASP). The energy of the ground state was obtained with Perdew-Burke-Ernzerhof (PBE) exchange-correlation potential. In order to represent core electrons, the projected augmented wave (PAW) potential was used with energy cutoff $500 \mathrm{eV}$. Ce atoms show strong localization of $4 f$-states which cannot be treated within pure DFT method. To overcome this, $4 f$-electrons were additionally described in terms of the Dudarev approach within DFT+U correction ${ }^{41}$ with Hubbard parameter $\mathrm{U}=4.5 \mathrm{eV}$, which was proposed before $^{36,42,43}$. Cubic $\mathrm{Fm} \overline{3} \mathrm{~m}$ crystal structure (225 space group) was used to represent atomic arrangement of fluorite $\mathrm{CeO}_{2}$. For the lattice and optical parameters calculation for the pure $\mathrm{CeO}_{2}$, a unit cell containing 12 atoms (4 $\mathrm{Ce}$ and $8 \mathrm{O}$ atoms) was considered.

The effect of different doping atoms on the electronic structure was considered for a $(2 \times 2 \times 2)$ supercell. Atoms of $\mathrm{Zr}$, Ti, Sn and $\mathrm{Si}$ were considered as extrinsic defects in $\mathrm{CeO}_{2}$ in an amount of 1 per supercell which gave the relation of doping to $\mathrm{Ce}$ atoms $\mathrm{X} /(\mathrm{Ce}+\mathrm{X})(\mathrm{X}-$ doping atom) equal to 3.1\%. Following the $\mathrm{DFT}+\mathrm{U}$ approach, $d$ electrons of $\mathrm{Zr}$, Ti, Sn were represented respectively with $\mathrm{U}$-values of $2.0 \mathrm{eV}^{26,44}, 4.2 \mathrm{eV}^{45}, 4.0 \mathrm{eV}^{46,47}$, as implemented in previous theoretical works. Spin-polarization was enabled whenever it was necessary.

For precise calculation of the optical parameters and the band structure, a hybrid potential was used. The DFTHF mixing parameter $a_{\text {mix }}$ was determined based on the static dielectric constant ${ }^{48}$ :

$$
a_{\text {mix }}=\frac{1}{\varepsilon_{\infty}}
$$

where the dielectric constant $\varepsilon_{\infty}$ was computed within the PBE potential.

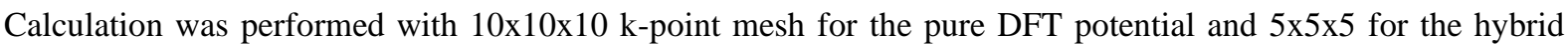
potential, using the Monkhorst-Pack scheme ${ }^{49}$. Convergence threshold for the system energy was chosen as $10^{-7}$ $\mathrm{eV}$ for the electronic steps and $10^{-6} \mathrm{eV}$ for the ionic steps.

The imaginary part of the optical dielectric function $\varepsilon_{2}$ has been derived from DFT results by summing the interband transitions from occupied to unoccupied states for energies much higher than those of phonons. The real part of the dielectric function $\varepsilon_{1}$ is calculated using the Kramers-Kronig transformation. The knowledge of both the real and imaginary parts of the dielectric tensor allows one to calculate other optical parameters. In this paper, we present and analyze the reflectivity $R(h v)$, the absorption coefficient $\alpha(h v)$, the refractive index $n(h v)$, and the extinction coefficient $k(h v)$. More details about the optical calculations were discussed elsewhere ${ }^{50,51}$.

\subsection{Defect formation energy}

The vacancy formation energy was evaluated with following expression ${ }^{52}$ :

$$
E_{f}\left(V_{O}^{q}\right)=E_{t o t}\left(V_{O}^{q}\right)-E_{\text {tot }}(\text { bulk })+\mu_{O}+q\left(E_{F}+E_{V B M}\right)
$$

where $E_{\text {tot }}\left(V_{O}^{q}\right)$ is the energy of the defect structure, $E_{\text {tot }}\left(\right.$ bulk) is the energy of the ideal structure, $\mu_{O}=$ $-4.93 \mathrm{eV}$ is the oxygen chemical potential, which was taking for the O-rich environment ${ }^{52}, q$ is the charge value (in case of charged vacancy), $E_{V B M}$ is the valence band maximum (VBM) energy, and $E_{F}$ is the Fermi level (above VBM).

\subsection{Water contact angle}

According to the Young equation, the contact angle can be expressed through surface tensions of different interfacial pairs:

$$
\cos \theta=\frac{\gamma_{S G}-\gamma_{S L}}{\gamma_{L G}}
$$

where $\gamma_{X Y}$ represents two-phases surface energy between solid, liquid or gas ( $\mathrm{S}, \mathrm{L}$ and $\mathrm{G}$, respectively). Water and ice are interchangeable due to their similarity in terms of surface energy, while atmospheric gas can be replaced by vacuum for the same reason. Solid-liquid interface is represented as adsorption of ice layers on a bare solid slab ${ }^{36}$ : 


$$
\gamma_{S L}=\gamma_{S G}+\frac{E_{a d s}^{i c e}}{A}
$$

and WCA is obtained in the form:

$$
\cos \theta=-\frac{E_{a d s}^{i c e}}{A \gamma_{L G}}
$$

Adsorption energy of the water bilayer in Eq. (4) can be rewritten in the form:

$$
E_{\text {ads }}^{i c e}=E_{\text {tot }}-\left(E_{S, \text { bulk }}+\gamma_{S G} A\right)-E_{\text {ice,bulk }}
$$

where $E_{\text {ice,bulk }}$ is the bulk energy for solid and ice.

Another approach proposed ${ }^{39}$ uses explicitly computed values for the surface tensions. For this, $\gamma_{S G}$ and $\gamma_{L G}$ are obtained from the energy difference for the bulk and slab materials per area unit. Surface tension of the SL interface can be expressed from the total energy of the slab containing both phases:

$$
E_{\text {tot }}=\gamma_{S G} A+\gamma_{S L} A+\gamma_{L G} A+E_{S, \text { bulk }}+E_{\text {ice,bulk }}
$$

where $A$ is the area of the considered supercell and $E_{S, b u l k}$ and $E_{i c e, b u l k}$ are the bulk energies for solid and ice.

Comparison of the two approaches described above gives that:

$$
\cos \theta_{1}=\cos \theta_{2}-1
$$

where $\theta_{1}$ is obtained from Eq. (4) and $\theta_{2}$ from Eq. (6). This relation points out that the first approach tends to overestimate the value of WCA due to exclusion of the water surface energy from the consideration.

Following previous studies on water adsorption on different surface ${ }^{39}$, the water molecule configuration has been determined using the $a b$ initio molecular dynamics method. Parameters of the molecular dynamics simulation included an Andersen thermostat at $200 \mathrm{~K}$ with a timestep of $0.1 \mathrm{fs}$ with total annealing time at $5 \mathrm{ps}$. The molecular dynamics step was followed by a standard DFT-optimization until reaching convergence in order to achieve equilibrium for the system.

\section{Results and Discussion}

\subsection{Materials properties for bulk $\mathrm{CeO}_{2}$}

Using the experimentally determined lattice parameters as input, structural optimization has been performed for $\mathrm{CeO}_{2}$ within $\mathrm{PBE}, \mathrm{PBE}+\mathrm{U}$ and hybrid functional calculations. The lattice parameter within $\mathrm{PBE}+\mathrm{U}$ potential equals to $5.495 \AA$ for stoichiometric $\mathrm{CeO}_{2}$, which is in good agreement with previous calculations ${ }^{52}$. The computed value of the optimized mixing parameter for the hybrid potential is determined from Eq. (1) as $a_{\text {mix }}=0.151$ and leads to a lattice constant of $5.417 \AA$ for ideal crystal, which corresponds well with the experimentally derived value $\left(\mathrm{a}_{\exp }=5.411 \AA\right)^{53}$. Lattice parameters for the equilibrium lattices of $\mathrm{M}_{0.25} \mathrm{Ce}_{0.75} \mathrm{O}_{2}$ are presented in Table 1 together with the experimentally determined values. Analysis of Table 1 shows that deviation of the calculated equilibrium volumes is $<6.5 \%$ of the experimentally determined values, which suggests that the theoretical calculations are in agreement with experimental observations. Furthermore, the symmetry of the optimized lattices for $\mathrm{CeO}_{2}$ is found to be the same as that of the corresponding experimentally determined one.

Table I - Lattice parameters for the doped $\mathrm{M}_{0.25} \mathrm{Ce}_{0.75} \mathrm{O}_{2}$ and pure $\mathrm{CeO}_{2}$ systems

\begin{tabular}{|c|c|c|c|c|c|c|}
\hline \multicolumn{2}{|c|}{ Doping atom } & $\mathrm{Zr}$ & $\mathrm{Sn}$ & $\mathrm{Ti}$ & $\mathrm{Si}$ & $\mathrm{Pure}^{\mathrm{CeO}_{2}}$ \\
\hline \multirow{2}{*}{$\begin{array}{c}\text { Lattice } \\
\text { parameter, } \AA\end{array}$} & DFT, PBE+U & 5.405 & 5.425 & 5.348 & 5.320 & 5.495 \\
\cline { 2 - 7 } & DFT, Hybrid & 5.338 & 5.350 & 5.284 & 5.245 & 5.440 \\
\cline { 2 - 7 } & Experimental & $5.352^{\mathrm{a}}$ & $5.388^{\mathrm{a}}$ & $5.396^{\mathrm{a}}$ & $5.406^{\mathrm{b}}$ & $5.411^{\mathrm{c}}$ \\
\hline
\end{tabular}

\footnotetext{
${ }^{a}$ Ref. ${ }^{54}$
}

${ }^{\mathrm{b}}$ Ref. 55

${ }^{b}$ Ref. ${ }^{53}$ 
The band structure calculated with the hybrid potential is shown in Fig. 1. It displays high localization of the $C e$ $4 f$ states with a narrow energy range that is in good agreement with results of Refs. ${ }^{56,57}$. A direct band gap of 3.07 $\mathrm{eV}$ corresponds to experimental values found in the range $3.03-3.57 \mathrm{eV}^{23,58-60}$ and is consistent with the previously reported range 3.0-3.25 eV for different hybrid potentials ${ }^{61}$. The fundamental band gap of $3.00 \mathrm{eV}$ between $\mathrm{X}(0$, $1 / 2,1 / 2)$ and $\mathrm{L}(1 / 2,1 / 2,1 / 2) \mathrm{k}$-points points to an indirect band gap nature for $\mathrm{CeO}_{2}$. The small deviation between indirect and direct band gaps is observed due to the strong localization of $4 f$ states, which implies a high sensitivity of the final band structure to calculation parameters, including the number of k-points and the meshing scheme.

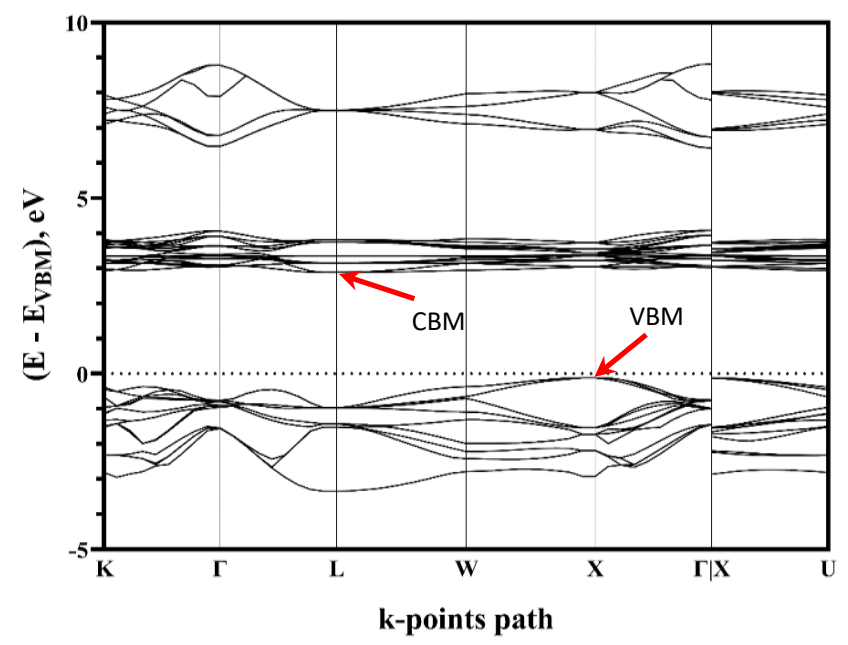

Fig. 1 Band structure of $\mathrm{CeO}_{2}$ calculated with hybrid potential $(\mathrm{a}=0.14)$. Valence band maximum (VBM) is placed to $0 \mathrm{eV}$.

\subsection{Intrinsic point defects}

Intrinsic defects in $\mathrm{CeO}_{2}$ are mostly represented by oxygen vacancies due to the relatively low formation energy that governs the defect equilibrium concentration at non-zero temperature. In order to investigate the influence of the oxygen vacancy on the electronic structure of $\mathrm{CeO}_{2}$, a vacancy has been created in the $2 \times 2 \times 2$ supercell of $\mathrm{CeO}_{2}$. The ideal supercell contains 96 atoms and the oxygen vacancy pushes the system to non-stoichiometric $\mathrm{CeO}_{2-\delta}$, where $\delta \approx 0.031$. For the charged defects, we assume charge delocalization and exclude the formation of the polarons from the consideration, even though they show slightly reduced formation energies for the pure system $^{52}$. The dependence of the formation energy for the different charge states of the oxygen vacancy $\left(\mathrm{V}_{\mathrm{O}}\right)$ on the Fermi level is plotted in Fig. 2 (a). The analysis shows that $\mathrm{V}_{\mathrm{O}}{ }^{+2}$ occurs to be more stable at a Fermi level range of $1.26-1.7 \mathrm{eV}$ and $0.14-1.7 \mathrm{eV}$ for oxygen poor and oxygen rich conditions, respectively. The $(+2 / 0)$ transition at $1.7 \mathrm{eV}$ indicates the vacancy induced deep state levels in the band gap which behave as negative-U center. It suggests that it is preferale for the oxygen vacancy to release/accept two electrons at the same time rather than one electron. Since $\mathrm{CeO}_{2}$ is an insulator, probability of accepting two electrons at the same time is too small. Thus, one cannot expect enhanced recombination activity from the anion vacancy. It should be noticed also that the situation with $\mathrm{E}_{\mathrm{f}}^{\mathrm{v}}(\mathrm{O})<0$ for positively charged vacancies leads to violation of the electroneutrality principle because negatively charged point defects cannot be created in enough quantities to compensate them. Singly charged vacancy does not appear as the most stable condition at any Fermi level. 

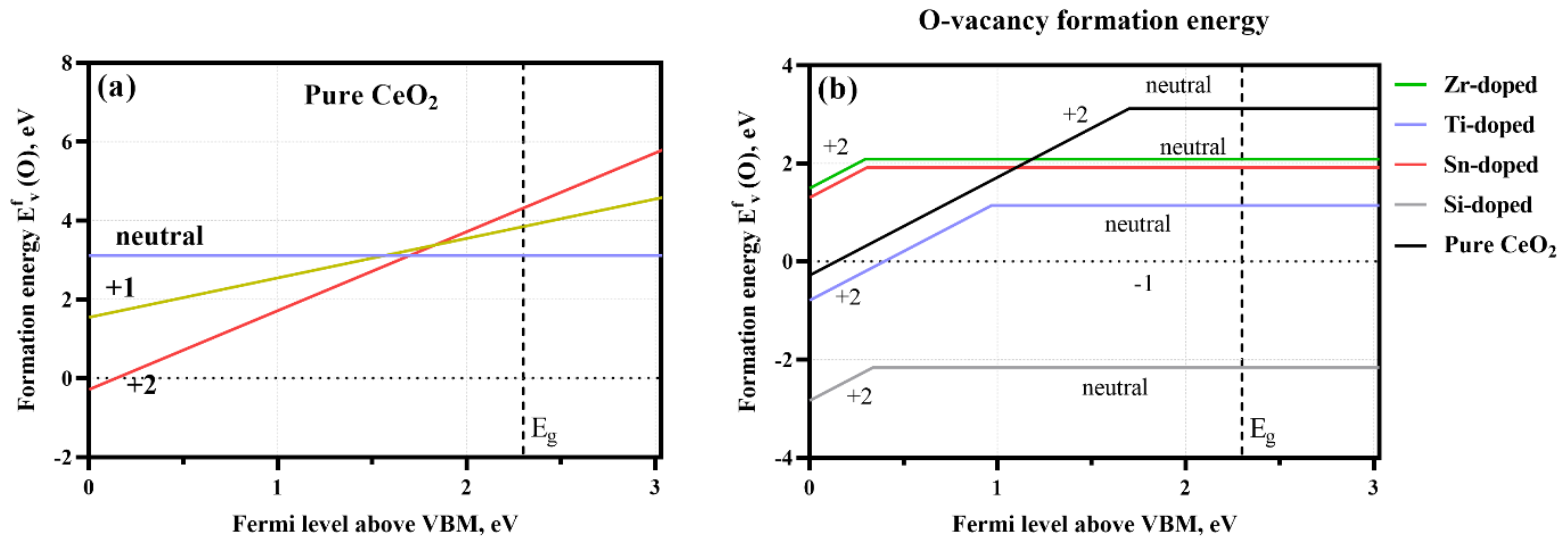

Fig 2. Dependence of oxygen vacancy formation energy on $\mathrm{E}_{\mathrm{F}}$ for (a) undoped and (b) doped $\mathrm{CeO}_{2}$ for $\mathrm{O}$-rich conditions.

\subsection{Extrinsic defects}

Fig. 3 displays the total density of states (TDOS) corresponding to $\mathrm{CeO}_{2}$ and $\mathrm{Ce}_{1-\mathrm{x}} \mathrm{M}_{\mathrm{x}} \mathrm{O}_{2}$ systems $(\mathrm{M}=\mathrm{Zr}, \mathrm{Ti}, \mathrm{Sn}$, $\mathrm{Si} ; \mathrm{x}=0.031$ ). Table II shows the defect formation energy $\mathrm{E}_{\mathrm{def}}{ }_{\mathrm{f}}^{\mathrm{f}}$, M-O bond length, and $O 2 p-C e 4 f$ band gap calculated within DFT $+\mathrm{U}$ approach in the current study. Introduction of the impurity cation into the fluorite structure causes strong crystal distortion around the defect with local rearrangement of the neighboring atoms. This change can be characterized qualitatively by the defect formation energy. Analysis of the doped structures shows a shift of the neighboring oxygen atoms towards the defect site where the Ce cation should be in an ideal lattice. The shift is caused by the smaller ionic radius compared to the $\mathrm{Ce}^{4-}$ cation. Due to the ability of $\mathrm{Zr}$ to form fluorite-arranged bounds with oxygen (implemented in cubic zirconia ${ }^{62}$ ), the local atomic arrangement around the solute keeps the fluorite symmetry of the ideal crystal even for the heavily doped systems ${ }^{63}$. This consistency of the lattices leads to a low defect formation energy $\mathrm{E}_{\mathrm{def}}{ }^{\mathrm{f}}$ for the $\mathrm{Zr}$-atom. The following reduction of the atomic radius for the impurities causes increaed defect formation energies. Moreover, the different $\mathrm{Me}-\mathrm{O}$ chemical bonds coordination for the doping atom oxides (6-, 6-, and 4-coordinated in $\mathrm{TiO}_{2}, \mathrm{SnO}_{2}$ and $\mathrm{SiO}_{2}$ respectively) causes rearrangement of the electronic structure that additionally increases value of $E_{\text {dop }}{ }^{f}$.

Using the DFT+U approach, we reproduce the general conclusions on the effect of doping atoms in $\mathrm{CeO}_{2}$. The high density of states located 2-3 eV above VBM is caused by strong localized $4 f$-states which remain unoccupied in the defect-free condition. Incorporation of the doping atom in the lattice modifies TDOS [Fig. 3, b-e]. All the considered extrinsic defects form additional deep levels in the gap between the $\mathrm{O} 2 \mathrm{p}-\mathrm{Ce} 5 \mathrm{~d}$ levels, which are attributed to $d$-states in $\mathrm{Zr}$, Ti, $\mathrm{Sn}$ systems and to $3 s$-states in $\mathrm{Si}$-doped $\mathrm{CeO}_{2}$. Another effect of the extrinsic defects is the slight shrinking of the $\mathrm{O} 2 \mathrm{p}$-Ce $4 f$ band gap, as shown in Table II. It should be noted that the yttrium-doped system possesses a metallic behavior with unoccupied $O 2 p$ states in the valence band, which most likely were created due to the reduced number of valence electrons of the extrinsic defect.

Table II - Characteristics of $\mathrm{Ce}_{1-\mathrm{x}} M_{\mathrm{x}} \mathrm{O}_{2}$ system $(M=\mathrm{Zr}$, Ti, Sn, $\mathrm{Si}, \mathrm{x}=0.031)$.

\begin{tabular}{|c|c|c|c|c|c|}
\hline Impurity & Undoped $\mathrm{CeO}_{2}$ & $\mathrm{Zr}$ & $\mathrm{Ti}$ & $\mathrm{Sn}$ & $\mathrm{Si}$ \\
\hline $\begin{array}{c}\text { Defect formation } \\
\text { energy E Edop }\end{array}$ & - & 0.06 & 4.69 & 4.91 & 6.92 \\
\hline Me-O bond length, $\AA$ & 2.38 & $2.29-$ & 2.24 & 2.26 & 2.16 \\
\hline $\begin{array}{c}\text { O2p-Ce4f band gap, } \\
\mathrm{eV}\end{array}$ & 2.38 & 2.18 & 2.10 & 2.09 & 1.95 \\
\hline
\end{tabular}




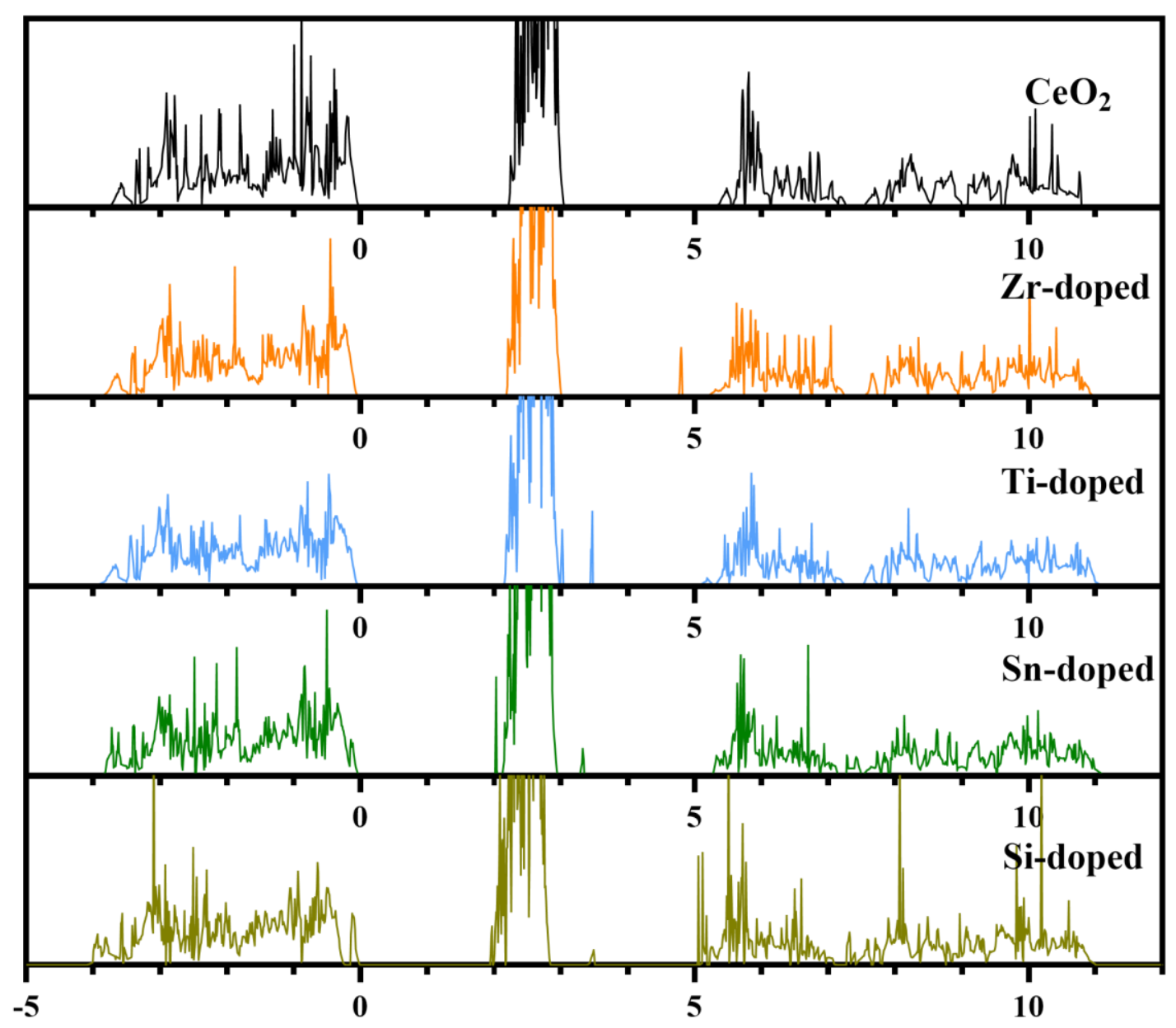

$\left(\mathbf{E}-\mathbf{E}_{\mathrm{VBM}}\right), \mathrm{eV}$

Figure 3. - TDOS for $\mathrm{CeO}_{2}$ and $\mathrm{Ce}_{1-\mathrm{x}} M_{\mathrm{x}} \mathrm{O}_{2}$ systems $(M=\mathrm{Zr}$, Ti, $\mathrm{Sn}, \mathrm{Si}$; $\mathrm{x}=0.0312)$

\subsection{Optical properties}

Figure 4 displays the calculated optical spectra for different doped systems. Besides using the PBE $+U$ approach we performed calculations within the hybrid potential which gives a reasonable estimation for the absorption edge value. To determine the influence of different extrinsic defects on the optical properties of $\mathrm{CeO}_{2}$, optical band gaps were estimated with Tauc plot reconstructed from the theoretically obtained spectra [Table I]. To save computational cost, the DFT+HF method was used for the single unit cell which corresponds to a high concentration of the dopants, i.e., $25 \%$ of all cations. It is seen from Table III that the PBE+U approach underestimates the optical band gap due to lack of the exact exchange in the description of the electrons behavior ${ }^{33}$. Dependence of the refractive index on the absorption coefficient through the Kramers-Kronig relation also predicts the analogous shift for the refractive index peak towards higher photon energies. It is clearly seen from [Fig. 4 (a, c, e, g)] that change the potential from $\mathrm{PBE}+\mathrm{U}$ to hybrid is followed by the main peak migration for refractive index from energy $\sim 2.6 \mathrm{eV}$ to $\sim 3.25 \mathrm{eV}$ for pure and doped ceria. 

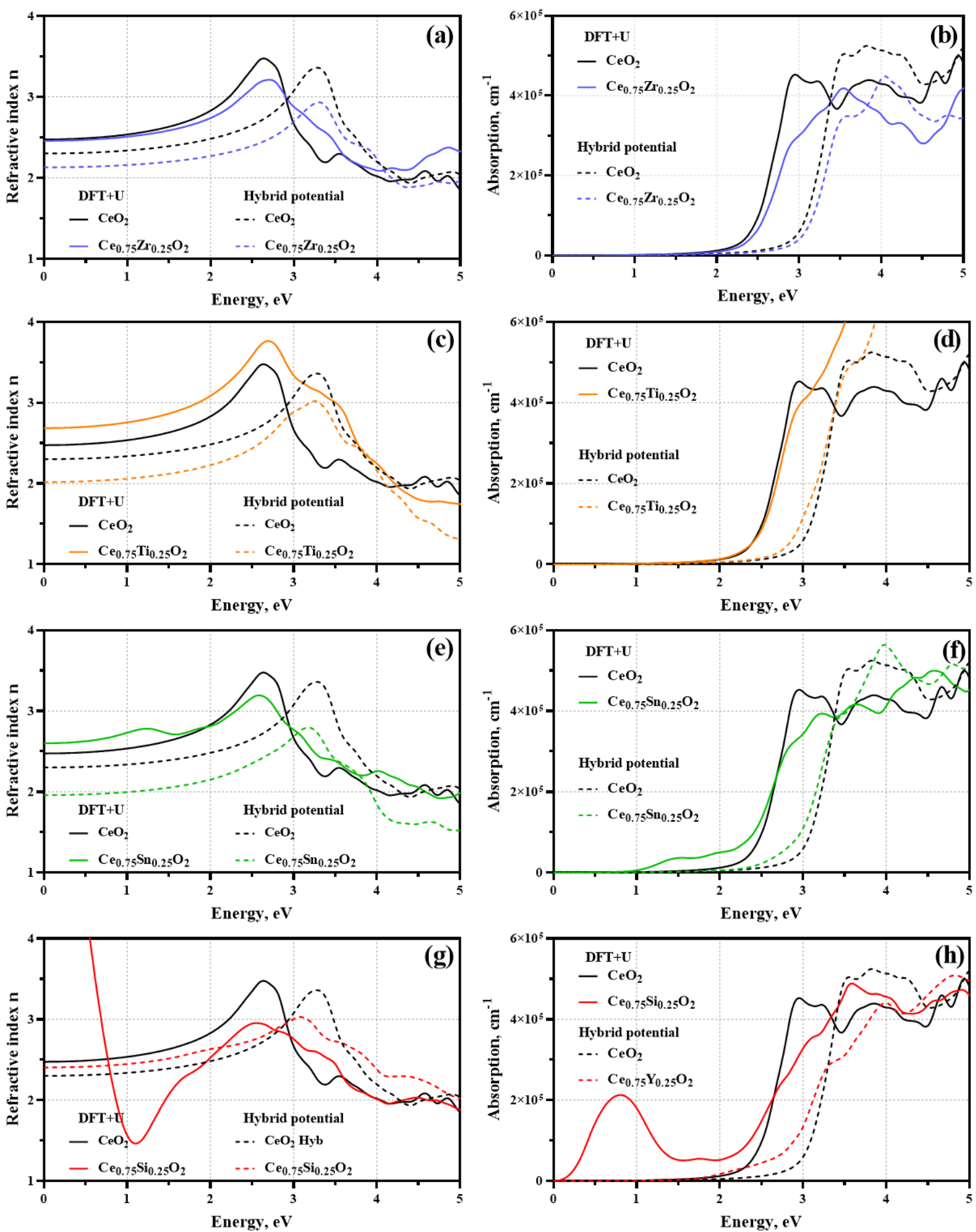

Fig. 4 - Calculated optical properties spectra for $\mathrm{Me}_{0.25} \mathrm{Ce}_{0.75} \mathrm{O}_{2}$ systems: (a, c, e, g) - refractive index; (b, d, f, h) - absorption coefficient for $\mathrm{Me}=\mathrm{Zr}, \mathrm{Ti}, \mathrm{Sn}, \mathrm{Si}$, respectively

Control of the optical properties remains one of the possible methods to enhance the practical usage of the material. The calculated optical properties of showed in Fig. 4 suggest the practical applications where such doping can be used to enhance the efficiency of ceria as functional material. Presence of $\mathrm{Zr}$ atoms in $\mathrm{CeO}_{2}$ increases the optical 
band gap and reduces the refractive index in the visible part of the electromagnetic spectrum, according to both DFT $+\mathrm{U}$ and hybrid potential calculations [Fig. $4(\mathrm{a}-\mathrm{b})$ ]. This is consistent with the previously reported results within the DFT+U framework ${ }^{26}$ and is confirmed by experiments ${ }^{28}$. The increased band gap of the doped system compared to pure $\mathrm{CeO}_{2}$, implies lower absorbance in the visible part of the electromagnetic spectrum while lower refractive index indicates less reflectance of visible light from the interface of the material with air. $\mathrm{Zr}$-doped $\mathrm{CeO}_{2}$ thus possesses higher transmittance than $\mathrm{CeO}_{2}$, which is beneficial for transparent optical coatings.

Incorporation of $\mathrm{Ti}$ atoms into $\mathrm{CeO}_{2}$ causes blue shift of the adsorption edge towards lower photon energies and reduces the refractive index, which enhances suppression of light reflection according to optical spectra obtained with the hybrid potential [Fig. 4 (c-d)]. This contradicts the results within the DFT+U approach that suggests increasing of the refractive index in the visible part of the electromagnetic spectrum ${ }^{28}$. Based on the DFT $+U$ approach, incorporation of $\mathrm{Sn}$ or $\mathrm{Si}$ doping atoms in $\mathrm{CeO}_{2}$ leads to continuous reduction of the adsorption edge energy while keeping the refractive index around that corresponding to undoped $\mathrm{CeO}_{2}$ in case of doping with $\mathrm{Sn}$ [Fig. 4 (e-f)] or slightly increasing it for the visible light in case of doping with Si [Fig. 4 (g-h)]. This contradicts to the results coming out from the calculations within the hybrid potential demonstrating a decrease of the refraction index over the visible part of the electromagnetic spectrum for Sn. Band gap change reduction for doping with $\mathrm{Sn}^{31}$ and $\mathrm{Si}^{32}$ qualitatively corresponds well with experimental observations. The theoretical analysis of the optical properties suggests enhancement of the visible and $\mathrm{UV}$-light absorption of $\mathrm{CeO}_{2}$ doped by $\mathrm{Ti}$, $\mathrm{Si}$ and $\mathrm{Sn}$. Such improvement in the light harvesting properties can be relevant for application in photocatalytic processes.

Table III. Optical band gaps for the doped systems $\mathrm{Ce}_{0.75} M_{0.25} \mathrm{O}_{2}$ obtained from the calculated permittivity spectra

\begin{tabular}{|c|c|c|c|}
\hline \multirow{2}{*}{ Doping atom } & \multicolumn{3}{|c|}{ Optical band gap E ${ }^{\text {opt }}(\mathrm{eV})$} \\
\cline { 2 - 4 } & PBE+U & HSE06 & Experiment \\
\hline $\mathrm{Zr}$ & 2.4 & 3.15 & 3.30 \\
\hline $\mathrm{Sn}$ & 1.0 & 2.8 & $2.72^{[31]}$ \\
\hline $\mathrm{Ti}$ & 2.3 & 2.75 & 3.00 \\
\hline $\mathrm{Si}$ & 0 & 2.5 & $2.61^{32}$ \\
\hline Pure $\mathrm{CeO}_{2}$ & 2.3 & 3.05 & $3.03-3.57$ Refs $^{23,58-60}$ \\
\hline
\end{tabular}

\subsection{Vacancy-impurity complex}

To investigate the effect of different tetravalent doping atoms on oxygen vacancy formation energy, an oxygen vacancy was introduced to the $2 \times 2 \times 2$ doped supercell with doping level at $3.1 \%$. Due to inequality of the different anions around the extrinsic defect, it is necessary to consider vacancy at 4 different nearest neighbors' positions (Fig. 5) ${ }^{64}$.

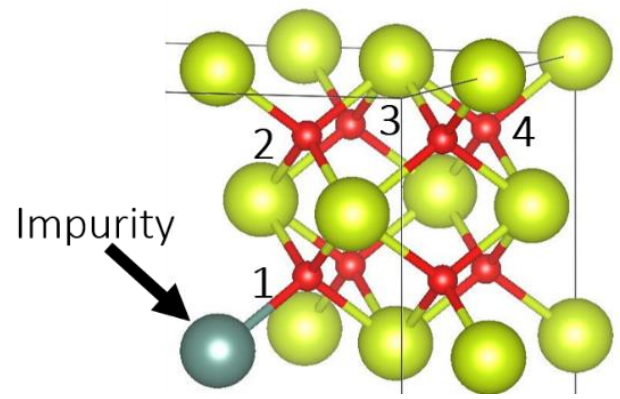

Fig. 5 - Nearest neighbors' position of anions for the cation doping atom

Formation energies of the oxygen vacancy for the different doped systems are calculated [Table IV]. The results for $\mathrm{Zr}$-, Ti-, and $\mathrm{Sn}$-doped systems are consistent with previous theoretical results and follow the general trend of different doping atoms to reduce $\mathrm{E}_{\mathrm{f}}^{\mathrm{v}}$ for the NN1 position ${ }^{64}$. According to the Boltzmann law, this reduction of the formation energies facilitates vacancies creation. The increased impurity concentration stimulates ionic conductivity of such systems, except for $\mathrm{Si}$, which is confirmed experimentally ${ }^{65,66}$. Analysis of the different positions for the vacancies (Table IV) points out the preferable creation of the oxygen vacancies near the doping atom. This position of the vacancy allows to minimize the strain energy caused by the incorporated extrinsic defect. 
Negative values for the formation energies of $\mathrm{Si}$-doped $\mathrm{CeO}_{2}$ points to strong rearrangement of the nearest impurity neighbors due to high mismatch in the atomic radii and different coordination of $\mathrm{Me}-\mathrm{O}$ bounds in $\mathrm{CeO}_{2}$ and $\mathrm{SiO}_{2}{ }^{67}$. The change of the local atomic structure causes interruption in the lattice order and produces heterogeneous inclusion which can be considered as a nucleus of a secondary phase. Negative formation energies thus can be interpretated as a sign of a high solubility of the atom in the host lattice. Represented as an additional obstacle for oxygen ions to migrate, the Si-based secondary phases in $\mathrm{CeO}_{2}$ reduce the ionic conductivity of $\mathrm{CeO}_{2}$ 68 .

Table IV. Oxygen vacancy formation energy in neutral state for oxygen-rich conditions for doped $\mathrm{CeO}_{2}$ with different impurities.

\begin{tabular}{|c|c|c|c|c|c|}
\hline & \multicolumn{5}{|c|}{$E_{f}^{v}(\mathrm{O}), \mathrm{eV}$} \\
\cline { 2 - 5 } & $\mathrm{Zr}$ & $\mathrm{Si}$ & $\mathrm{Sn}$ & $\mathrm{Ti}$ & \multirow{2}{*}{3.12} \\
\cline { 2 - 5 } NN1 & 2.08 & -2.16 & 1.92 & 1.17 & \\
\hline NN2 & 3.17 & -0.63 & 3.44 & 2.94 & \\
\hline NN3 & 3.07 & -0.21 & 3.47 & 2.05 & \\
\hline NN4 & 3.08 & -1.45 & 2.61 & 2.32 & \\
\hline
\end{tabular}

Dependence of the O-vacancy formation energy on Fermi level for the doped $\mathrm{CeO}_{2}$ is depicted in Fig. 2, b. Doped $\mathrm{CeO}_{2}$ possesses negative- $\mathrm{U}$ center behavior for the oxygen vacancy which is described as preferrable formation of the neutral defect over the single charged one. The transition between +2 and neutral charge states for the vacancy occurs at the levels $0.30 \mathrm{eV}, 0.97 \mathrm{eV}, 0.31 \mathrm{eV}, 0.34 \mathrm{eV}$ above VBM for Zr-, Ti-, Sn- and Si-doped systems, respectively. These transition energies indicate a position of the double deep level within the $2 p-4 f$ bandgap.

\subsection{Wettability}

For the current study, low index surfaces $\left(100,110\right.$ and 111) of pure and doped $\mathrm{CeO}_{2}$ were considered. In order to obtain water contact angle for the specific surface, total energy has been computed for a $\mathrm{CeO}_{2}$ slab with water molecules on it [Fig. 6]. The $\mathrm{CeO}_{2}$ slabs terminated by oxygen atoms were constructed from fluorite $\mathrm{CeO}_{2}$ by its cleavage along specific crystallographic planes. The obtained slab was expanded with formation of a supercell (2x2) along axes parallel to the surface for a better fit of the water molecules and three unit cells in depth. Impurity atoms for doped systems were placed in the surface layer to maximize their effect on surface energy and water molecules configuration. Water slab in the form of ice crystal consisted of double bilayer that fully covered the surface of $\mathrm{CeO}_{2}$. The number of water molecules in a layer for the proposed model corresponded to the number of the closest cation atoms in the $\mathrm{CeO}_{2}$ slab, which caused significant distortion in the ice structure. For the ideal hexagonal ice structure on undoped- $\mathrm{CeO}_{2}$ the total change in the water molecule density was $9.9 \%,-154.0 \%$ and $22.3 \%$ for (100), (110) and (111) surfaces, respectively (negative value indicates crystal expansion).

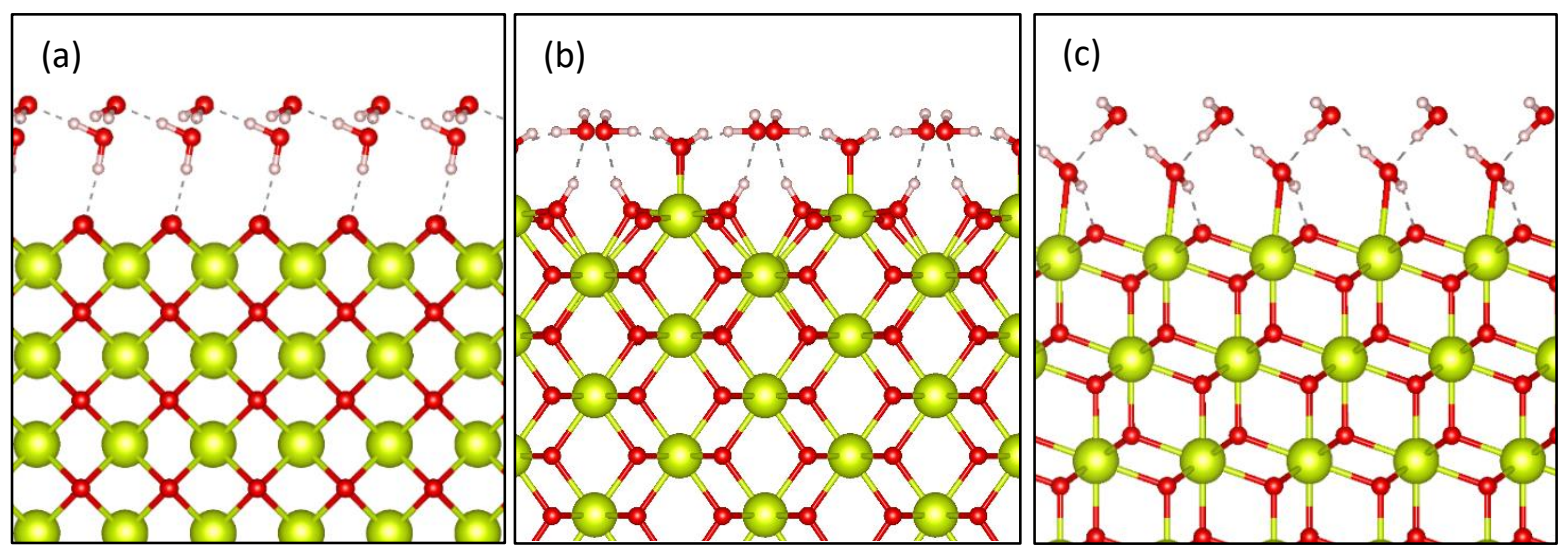

Fig. $6-\mathrm{CeO}_{2}$-water slabs after relaxation for different low-index surfaces of ceria: (a) - (100); (b) - (110); (c) (111) 
Calculated contact angles for the pure ceria are summarized in the Table V as compared with results of Refs. ${ }^{36,39,40}$

Table V - Calculated water contact angles for low-index $\mathrm{CeO}_{2}$ planes

\begin{tabular}{|c|c|c|c|c|c|}
\hline \multirow{3}{*}{ Plane index } & \multicolumn{5}{|c|}{ Computed WCA, ${ }^{\circ}$} \\
\hline & \multicolumn{2}{|c|}{ Current study } & \multirow{2}{*}{ Fronzi et al. ${ }^{39}$} & \multirow{2}{*}{$\begin{array}{c}\text { Carchini et } \\
\text { al. }^{36}\end{array}$} & \multirow{2}{*}{ Experiment $^{40}$} \\
\hline & Eq. (6) & Eq. (4) & & & \\
\hline (100) & 19.36 & 93.24 & 93.91 & - & 36.5 \\
\hline (110) & 0 & 65.10 & 64.09 & - & 41.1 \\
\hline (111) & 52.71 & 113.21 & 112.53 & 99.9 & 57.1 \\
\hline
\end{tabular}

The analysis shows that the approach based on adsorption energy provides better agreement with Refs. ${ }^{36,39}$ and suggests a hydrophobic behavior of $\mathrm{CeO}_{2}$ for (100) and (111) planes. However, WCA obtained from Eq. (6) reveal hydrophilicity of $\mathrm{CeO}_{2}$ regardless the plane index. Experimental results for an atomically flat surface of $\mathrm{CeO}_{2}$ with different indexes ${ }^{40}$ show good correspondence of the approach for (111) plane, while for (110) and (100) the theoretically determined CA tends to be underestimated. Such mismatch can be partly explained by fast deposition of airborne hydrocarbons which rapidly increase hydrophobicity of rare-earth oxides during exposure to atmospheric air and in vacuum systems. Multiple studies show the ability of $\mathrm{CeO}_{2}$ to reverse the wetting properties after annealing ${ }^{21}$ or plasma treatment ${ }^{19}$ which confirms the idea of hydrocarbons depositing onto the surface when exposed to air.

To estimate the effect of different dopants on the wetting properties of $\mathrm{CeO}_{2}$, one of the cations in the solid slab was replaced by an extrinsic defect. The position of the doping atom was chosen in the surface layer. Water contact angles were computed the for $\mathrm{Ce}_{1-\mathrm{x}} \mathrm{Me}_{\mathrm{x}} \mathrm{O}_{2}$ (111) plane with one replaced atom, i.e., with surface concentration of the doping atom at $25 \%$ (at.). The results obtained are summarized in Table VI.

Table VI. WCA for doped $\mathrm{CeO}_{2}$ terminated by (111) surface

\begin{tabular}{|c|c|c|c|c|c|}
\hline Doping atom & Undoped $\mathrm{CeO}_{2}$ & $\mathrm{Zr}$ & $\mathrm{Ti}$ & $\mathrm{Sn}$ & $\mathrm{Si}$ \\
\hline WCA (Eq. (4)), ${ }^{\circ}$ & 113.21 & 108.48 & 101.73 & 107.01 & 88.24 \\
\hline WCA (Eq. (6)), $^{\circ}$ & 52.71 & 46.92 & 37.14 & 44.98 & 0 \\
\hline $\mathrm{d}_{\text {Me-O }}, \AA$ & 2.375 & 2.175 & 1.953 & 2.121 & 1.695 \\
\hline Surface energy, J/m & 0.51 & 0.63 & 1.47 & 2.21 & 1.53 \\
\hline
\end{tabular}

The analysis shows that doping reduces WCA for $\mathrm{CeO}_{2}$. Tuning of the wettability originating from the geometry of the surface was previously reported and attributed to the mismatch between the ice and $\mathrm{CeO}_{2} \mathrm{lattices}^{36}$. According to that, the ice bilayer experiences slight squeezing during adsorption on the surface which leads to the minimization of the interaction energy. Further reduction of the metal-to-metal distance is expected to enhance the mismatch between oxide surface and water layer and to increase WCA. Besides the metal-to metal distance, an important characteristic which governs the wetting behavior, is distortion of the oxygen sublattice close to the doping atom. Doping with the metal impurity causes local rearrangement of the oxide anions by changing the metal-to-oxygen distance. Such rearrangement creates a local deviation from the flat condition [Fig. 7, a], which influences the water molecules from the first bilayer. The larger deviation in the metal-oxygen length $\mathrm{d}_{\mathrm{Me}-\mathrm{O}}$ for the doped system from $\mathrm{Ce}-\mathrm{O}$ bond in pure $\mathrm{CeO}_{2}$ implies creation of a bigger roughness in the atomic scale and tighter connection between water and oxide surface. Assuming the same type of chemical bond between impurity and oxygen as for $\mathrm{Ce}$, it is found that the metal-oxygen length $\mathrm{d}_{\mathrm{Me}-\mathrm{O}}$ depends on the atomic radius of the impurity atom. This allows to see the correlation between doping tetravalent atom in the $\mathrm{CeO}_{2}$ surface and intrinsic wetting properties of such system [Fig. 7, b]. It should be noted that the surface energy, i.e., the relaxation energy of the surface atoms over the area unit, can vary between $0.51 \mathrm{~J} / \mathrm{m}^{2}$ for the pure $\mathrm{CeO}_{2}$ and $2.21 \mathrm{~J} / \mathrm{m}^{2}$ for the Sn-doped 
$\mathrm{CeO}_{2}$ [Table VI]. This change, however, is not reflected much in the variation of WCA values, assuming lower dependence of this parameter on the tension characteristic of the surface in the absence of water.
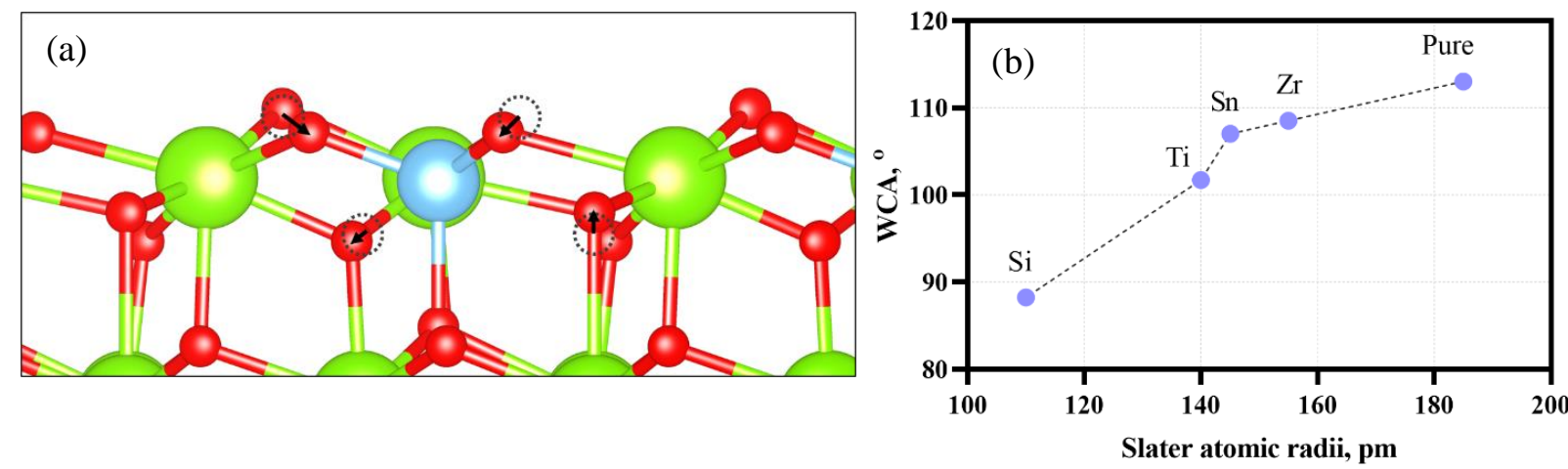

Fig. 7 (a) - displacement of the oxygen atoms closest to the doping atom in the oxide slab surface; $b$ Dependence of water contact angle of doped $\mathrm{Ce}_{1-\mathrm{x}} \mathrm{Me}_{\mathrm{x}} \mathrm{O}_{2}(\mathrm{x}=0.05)$ (Eq. (4)) on the dopant atomic radii

The predicted intrinsic hydrophilicity of $\mathrm{CeO}_{2}$ remains fair only in absence of adsorbed species on the materials surface. During exposure to air, water contact angle for $\mathrm{CeO}_{2}$ increases up to $115^{\circ}$ for the flat surfaces, which was attributed to adsorption of airborne hydrocarbons ${ }^{19}$. The change in wetting properties caused by organic molecules depends on the ability of the surface to attract the organic molecules. The reason why one material possesses a higher hydrocarbon content on its surface after air exposure compared to another material still remains unclear. The possible explanation for $\mathrm{CeO} 2$ might be that its surface turns out to be more attractive to hydrocarbons due to a decreased adsorption energy for the organic molecules. The proof of this statement requires a systematic experimental and theoretical consideration of the adsorption ability of $\mathrm{CeO}_{2}$ and hydrophilic oxides (for example, $\mathrm{Al}_{2} \mathrm{O}_{3}$ ) for different types of organic substances. The main requirement for the higher concentration of hydrocarbon molecules on the surface implies the abundance of vacant positions where those molecules can be adsorbed. Such places on the surface might be represented by, for example, oxygen vacancies or adsorbed hydroxyl groups ${ }^{38}$. In this work, the higher oxygen vacancy concentration was predicted due to decreased formation energy for that defect in the doped system. Oxygen vacancies on the surface are attributed to the ability to dissociate water ${ }^{69}$. The $\mathrm{OH}^{-}$-groups formed by that process then become adsorbed on the surface by filling the vacancy sites. These groups can be used for the attraction of different hydrocarbon molecules and their derivatives, which are present in air or in low-vacuum systems, switching the material to a hydrophobic state ${ }^{70,71}$. Absence of the oxygen vacancies in the doped $\mathrm{CeO}_{2}$ thus can provide additional spots for the organic molecules to be adsorbed on the surface, resulting in an increased repelling of the water molecules.

\subsection{Assessment of doping strategies for the glass industry applications}

In this section we would like to articulate ideas regarding practical usage of $\mathrm{CeO}_{2}$ doped by tetravalent atoms such as $\mathrm{Zr}, \mathrm{Ti}, \mathrm{Sn}$ and $\mathrm{Si}$ in the glass industry applications, based on the results presented in this work. As mentioned in the introduction, one of the limiting factors towards using $\mathrm{CeO}_{2}$ as a transparent layer remains its high refractive index and small band gap, which causes a reduction of the sunlight transmittance. The introduction of $\mathrm{Zr}$ in $\mathrm{CeO}_{2}$ reduces absorption and reflection of the visible light making such doped system more attractive for using as transparent layer. The ability of $\mathrm{Zr}$ to construct 8-coordinated fluorite-type chemical bonds with $\mathrm{O}$-atoms and atomic radius closer to that for $\mathrm{Ce}$ [Fig. 7 (b)] imply high solubility of $\mathrm{ZrO}_{2}$ in $\mathrm{CeO}_{2}$ without breaking the crystal symmetry ${ }^{63}$. This results in the lowest degradation in hydrophobicity of $\mathrm{CeO}_{2}$ [Table VI]. These factors define $\mathrm{Zr}$ as the preferable doping agent for $\mathrm{CeO}_{2}$ for applications in the glass industry. The high-level atomic rearrangement around solute for $\mathrm{Ti}-, \mathrm{Sn}-$, and $\mathrm{Si}$-doped systems leads to a significant change in the intrinsic wetting behavior of the $\mathrm{CeO}_{2}$ surface towards higher hydrophilicity. This also results in increased light absorption by the material in the visible part of the electromagnetic spectrum [Fig. 4]. That suggests the practical application of such solid solution as light-harvesting material which is capable of absorbing a wider range of photon energies than nondoped $\mathrm{CeO}_{2}$. The higher hydrophilicity, which causes lower WCAs, makes this group of dopants preferable for applications requiring attraction of water molecules to the surface. 


\section{Conclusion}

In this work we have considered the electronic structure of $\mathrm{CeO}_{2}$ doped with different atoms. With using the $\mathrm{PBE}+\mathrm{U}$ and hybrid potential, different properties were considered, including total density of states, refractive index, absorption, oxygen formation energy as well as hydrophobicity by means of water contact angle. The results suggest that $\mathrm{CeO}_{2}$ is a semiconductor with a fundamental band gap at $3.00 \mathrm{eV}$. It possesses intrinsic hydrophilic behavior of low-index surfaces which was showed with simulation of $\mathrm{CeO}_{2}$-water slabs. Doping of $\mathrm{CeO}_{2}$ with tetravalent atoms $(\mathrm{Zr}, \mathrm{Ti}, \mathrm{Sn}$ and $\mathrm{Si}$ ) modifies the electronic and optical properties in different ways, implying various possible applications of such doped systems. The solute atoms reduce the oxygen-formation energy and significantly change the configuration of the deep levels within the bandgap. It was shown that doping of $\mathrm{CeO}_{2}$ leads to the enhancement of the intrinsic hydrophilicity. Based on the theoretical approach, we conclude that $\mathrm{Zr}$-doped $\mathrm{CeO}_{2}$ appears to be most promising candidate material for use in the glass industry.

\section{Acknowledgement}

The computations have been performed by using the Norwegian Notur supercomputing facilities through the project nn4608k. The work has been supported by the INTPART project 309827 and M-ERA.net project 300107 funded by the Research Council of Norway. The authors thanks Dr. Elbruz Murat Baba and Dr. Ørnulf Nordseth (Institute for Energy Technology) for fruitful discussions.

\section{References}

1. Teh, L. P. et al. Recent progress in ceria-based catalysts for the dry reforming of methane: A review. Chem. Eng. Sci. 242, 116606 (2021).

2. Chang, K., Zhang, H., Cheng, M. J. \& Lu, Q. Application of Ceria in CO2 Conversion Catalysis. ACS Catal. 10, 613-631 (2020).

3. Montini, T., Melchionna, M., Monai, M. \& Fornasiero, P. Fundamentals and Catalytic Applications of CeO2-Based Materials. Chem. Rev. 116, 5987-6041 (2016).

4. Chueh, W. C., Hao, Y., Jung, W. \& Haile, S. M. High electrochemical activity of the oxide phase in model ceria-Pt and ceria-Ni composite anodes. Nat. Mater. 11, 155-161 (2012).

5. Wang, B. et al. Fast ionic conduction in semiconductor CeO2- $\delta$ electrolyte fuel cells. NPG Asia Mater. 11, (2019).

6. Cai, Y. et al. A Bulk-Heterostructure Nanocomposite Electrolyte of Ce0.8Sm0.2O2- $\delta$-SrTiO3 for LowTemperature Solid Oxide Fuel Cells. Nano-Micro Lett. 13, 1-14 (2021).

7. Magdalane, C. M., Kaviyarasu, K., Vijaya, J. J., Siddhardha, B. \& Jeyaraj, B. Photocatalytic activity of binary metal oxide nanocomposites of $\mathrm{CeO} 2 / \mathrm{CdO}$ nanospheres: Investigation of optical and antimicrobial activity. J. Photochem. Photobiol. B Biol. 163, 77-86 (2016).

8. Nadeem, M. et al. Green synthesis of cerium oxide nanoparticles (Ceo2 nps) and their antimicrobial applications: A review. Int. J. Nanomedicine 15, 5951-5961 (2020).

9. Anirban, S. \& Dutta, A. Revisiting ionic conductivity of rare earth doped ceria: Dependency on different factors. Int. J. Hydrogen Energy 45, 25139-25166 (2020).

10. Schmitt, R. et al. A review of defect structure and chemistry in ceria and its solid solutions. Chem. Soc. Rev. 49, 554-592 (2020).

11. Azimi, G., Dhiman, R., Kwon, H. M., Paxson, A. T. \& Varanasi, K. K. Hydrophobicity of rare-earth oxide ceramics. Nat. Mater. 12, 315-320 (2013).

12. Lundy, R. et al. Exploring the Role of Adsorption and Surface State on the Hydrophobicity of Rare Earth Oxides. ACS Appl. Mater. Interfaces 9, 13751-13760 (2017).

13. Hu, L. et al. A robust quasi-superhydrophobic ceria coating prepared using air-plasma spraying. J. Am. Ceram. Soc. 102, 1386-1393 (2019).

14. Zhang, M., Feng, S., Wang, L. \& Zheng, Y. Lotus effect in wetting and self-cleaning. Biotribology 5, 31-43 (2016)

15. Jeevahan, J., Chandrasekaran, M., Britto Joseph, G., Durairaj, R. B. \& Mageshwaran, G. Superhydrophobic surfaces: a review on fundamentals, applications, and challenges. J. Coatings Technol. Res. 15, 231-250 (2018).

16. Azimi, G., Kwon, H. M. \& Varanasi, K. K. Superhydrophobic surfaces by laser ablation of rare-earth oxide ceramics. MRS Commun. 7, 95-99 (2014). 
17. Khan, S., Azimi, G., Yildiz, B. \& Varanasi, K. K. Role of surface oxygen-to-metal ratio on the wettability of rareearth oxides. Appl. Phys. Lett. 106, 2-7 (2015).

18. Shi, Z., Shum, P., Zhou, Z. \& Li, L. K. Y. Effect of oxygen flow ratio on the wetting behavior, microstructure and mechanical properties of $\mathrm{CeO} 2-x$ coatings prepared by magnetron sputtering. Surf. Coatings Technol. 320, 333-338 (2017).

19. Preston, D. J. et al. Effect of hydrocarbon adsorption on the wettability of rare earth oxide ceramics. Appl. Phys. Lett. 105, 1-8 (2014).

20. Oh, I. K. et al. Hydrophobicity of rare earth oxides grown by atomic layer deposition. Chem. Mater. 27, 148-156 (2015).

21. Fu, S. P. et al. On the wetting behavior of ceria thin films grown by pulsed laser deposition. Appl. Phys. Lett. 110, $1-6(2017)$.

22. Prakash, S. et al. Intrinsic hydrophilic nature of epitaxial thin-film of rare-earth oxide grown by pulsed laser deposition. Nanoscale 10, 3356-3361 (2018).

23. Guo, S., Arwin, H., Jacobsen, S. N., Järrendahl, K. \& Helmersson, U. A spectroscopic ellipsometry study of cerium dioxide thin films grown on sapphire by rf magnetron sputtering. J. Appl. Phys. 77, 5369-5376 (1995).

24. Bueno, R. M., Martinez-Duart, J. M., Hernández-Vélez, M. \& Vázquez, L. Optical and structural characterization of r.f. sputtered CeO2 thin films. J. Mater. Sci. 32, 1861-1865 (1997).

25. Oh, T. S., Tokpanov, Y. S., Hao, Y., Jung, W. \& Haile, S. M. Determination of optical and microstructural parameters of ceria films. J. Appl. Phys. 112, (2012).

26. Tian, D. et al. Performance of cubic $\mathrm{ZrO} 2$ doped $\mathrm{CeO} 2$ : First-principles investigation on elastic, electronic and optical properties of Ce1-x ZrxO2. J. Alloys Compd. 671, 208-219 (2016).

27. Mastelaro, V. R., Briois, V., de Souza, D. P. F. \& Silva, C. L. Structural studies of a ZrO2-CeO2 doped system. J. Eur. Ceram. Soc. 23, 273-282 (2003).

28. Veszelei, M., Kullman, L., Granqvist, C. G., von Rottkay, N. \& Rubin, M. Optical constants of sputter-deposited Ti-Ce oxide and Zr-Ce oxide films. Appl. Opt. 37, 5993 (1998).

29. Peña-Rodríguez, O. et al. Optical properties of ceria-zirconia epitaxial films grown from chemical solutions. Mater. Chem. Phys. 138, 462-467 (2013).

30. Hartridge, A., Krishna, M. G. \& Bhattacharya, A. K. Structure and Optical Properties of Nanocrystalline Yttria Doped Ceria Thin Films. Int. J. Mod. Phys. B 12, 1573-1583 (1998).

31. Manibalan, G., Murugadoss, G., Thangamuthu, R., Kumar, M. R. \& Kumar, R. M. Facile synthesis of CeO2-SnO2 nanocomposite for electrochemical determination of L-cysteine. J. Alloys Compd. 792, 1150-1161 (2019).

32. Rani, N., Ahlawat, R. \& Goswami, B. Annealing effect on bandgap energy and photocatalytic properties of CeO2$\mathrm{SiO} 2$ nanocomposite prepared by sol-gel technique. Mater. Chem. Phys. 241, 122401 (2020).

33. Krcha, M. D. \& Janik, M. J. Examination of oxygen vacancy formation in Mn-Doped CeO2 (111) using DFT+U and the hybrid functional HSE06. Langmuir 29, 10120-10131 (2013).

34. Balestrieri, M. et al. Photoluminescence properties of rare earth ( $\mathrm{Nd}, \mathrm{Yb}, \mathrm{Sm}, \mathrm{Pr})$-doped $\mathrm{CeO} 2$ pellets prepared by solid-state reaction. J. Mater. Chem. C 3, 7014-7021 (2015)

35. Hong, T., Zhang, L., Chen, F. \& Xia, C. Structural and dielectric properties of the fluorite-type LaxCe1-xO2- $\delta$ ceramics. J. Phys. D. Appl. Phys. 218, 254-260 (2012).

36. Carchini, G., García-Melchor, M., Łodziana, Z. \& López, N. Understanding and Tuning the Intrinsic Hydrophobicity of Rare-Earth Oxides: A DFT+U Study. ACS Appl. Mater. Interfaces 8, 152-160 (2016).

37. Cornelius, S. et al. Oxyhydride Nature of Rare-Earth-Based Photochromic Thin Films. J. Phys. Chem. Lett. 10, 1342-1348 (2019).

38. Xu, P., Meng, G., Pershin, L., Mostaghimi, J. \& Coyle, T. W. Control of the hydrophobicity of rare earth oxide coatings deposited by solution precursor plasma spray by hydrocarbon adsorption. J. Mater. Sci. Technol. 62, 107$118(2021)$.

39. Fronzi, M., N. Assadi, M. H. \& Hanaor, D. A. H. Theoretical insights into the hydrophobicity of low index $\mathrm{CeO} 2$ surfaces. arXiv (2019).

40. Tam, J., Feng, B., Ikuhara, Y., Ohta, H. \& Erb, U. Crystallographic orientation-surface energy-wetting property 
relationships of rare earth oxides. J. Mater. Chem. A 6, 18384-18388 (2018).

41. Dudarev, S. L., Botton, G. A., Savrasov, S. Y., Humphreys, C. J. \& Sutton, A. P. Electron-energy-loss spectra and the structural stability of nickel oxide: An LSDA+U study. Phys. Rev. B 57, 1505-1509 (1998).

42. Cococcioni, M. \& De Gironcoli, S. Linear response approach to the calculation of the effective interaction parameters in the LDA+U method. Phys. Rev. B - Condens. Matter Mater. Phys. 71, 1-16 (2005).

43. Ganduglia-Pirovano, M. V., Da Silva, J. L. F. \& Sauer, J. Density-functional calculations of the structure of nearsurface oxygen vacancies and electron localization on CeO2(111). Phys. Rev. Lett. 102, 1-4 (2009).

44. Zhang, Y., Ji, V. \& Xu, K. W. The detailed geometrical and electronic structures of monoclinic zirconia. J. Phys. Chem. Solids 74, 518-523 (2013).

45. Morgan, B. J. \& Watson, G. W. A density functional theory + u study of Oxygen vacancy formation at the (110), (100), (101), and (001) surfaces of rutile TiO2. J. Phys. Chem. C 113, 7322-7328 (2009).

46. Villamagua, L. et al. Change in the electrical conductivity of $\mathrm{SnO} 2$ crystal from n-type to p-type conductivity. Chem. Phys. 452, 71-77 (2015).

47. Stashans, A., Puchaicela, P. \& Rivera, R. DFT study of chromium-doped SnO2 materials. J. Mater. Sci. 49, 29042911 (2014).

48. Marques, M. A. L., Vidal, J., Oliveira, M. J. T., Reining, L. \& Botti, S. Density-based mixing parameter for hybrid functionals. Phys. Rev. B - Condens. Matter Mater. Phys. 83, (2011).

49. Monkhorst, H. J. \& Pack, J. D. Special points for Brillouin-zone integrations. Phys. Rev. B 13, 5188-5192 (1976).

50. Karazhanov, S. Z., Ulyashin, A. G., Vajeeston, P. \& Ravindran, P. Hydrides as materials for semiconductor electronics. Philos. Mag. 88, 2461-2476 (2008).

51. Karazhanov, S. Z. et al. Phase stability, electronic structure, and optical properties of indium oxide polytypes. Phys. Rev. B - Condens. Matter Mater. Phys. 76, 1-13 (2007).

52. Zacherle, T., Schriever, A., De Souza, R. A. \& Martin, M. Ab initio analysis of the defect structure of ceria. Phys. Rev. B - Condens. Matter Mater. Phys. 87, 1-11 (2013).

53. Gerward, L. et al. Bulk modulus of $\mathrm{CeO} 2$ and $\mathrm{PrO} 2-\mathrm{An}$ experimental and theoretical study. J. Alloys Compd. 400, 56-61 (2005).

54. Baidya, T. et al. Understanding the anomalous behavior of Vegard's law in Ce 1-x M x O $2(\mathrm{M}=\mathrm{Sn}$ and Ti; $0<\mathrm{x}$ $\leq 0.5)$ solid solutions. Phys. Chem. Chem. Phys. 18, 13974-13983 (2016).

55. Reddy, B. M. \& Khan, A. Nanosized CeO2-SiO2, CeO2-TiO2, and CeO2-ZrO2 mixed oxides: Influence of supporting oxide on thermal stability and oxygen storage properties of ceria. Catal. Surv. from Asia 9, 155-171 (2005).

56. Ren, Y., Zhang, C., Liu, X., Bian, Z. \& Yin, Y. Elastic properties and electronic structure of transition metal atoms in $\mathrm{CeO} 2$ solid solution: First principle studies. Comput. Mater. Sci. 98, 459-465 (2015).

57. Xue, Y., Tian, D., Zeng, C., Fu, Y. \& Li, K. Effect of Fe doping concentration on photocatalytic performance of $\mathrm{CeO} 2$ from DFT insight into analysis. AIP Adv. 9, (2019).

58. Hogarth, C. A. \& Al-Dhhan, Z. T. Optical Absorption in Thin Films of Cerium Dioxide and Cerium Dioxide Containing Silicon Monoxide. Phys. status solidi 137, K157-K160 (1986).

59. Sundaram, K. B. \& Wahid, P. Optical Absoprtion in Ceirum Dioxide Thin Films. Phisic State Solids 161, 63-66 (1990).

60. Colis, S. et al. Extrinsic ferromagnetism in epitaxial Co-doped $\mathrm{CeO} 2$ pulsed laser deposited films. J. Appl. Phys. 108, (2010).

61. Graciani, J. et al. Comparative study on the performance of hybrid DFT functionals in highly correlated oxides: The case of $\mathrm{CeO} 2$ and $\mathrm{Ce} 2 \mathrm{O} 3$. J. Chem. Theory Comput. 7, 56-65 (2011).

62. Muhammad, I. D., Awang, M., Mamat, O. \& Shaari, Z. B. First-Principles Calculations of the Structural, Mechanical and Thermodynamics Properties of Cubic Zirconia. World J. Nano Sci. Eng. 04, 97-103 (2014).

63. Hirano, M., Miwa, T. \& Inagaki, M. Low-temperature direct synthesis of nanoparticles of fluorite-type ceriazirconia solid solutions by 'Forced cohydrolysis' at $100^{\circ}$ C. J. Solid State Chem. 158, 112-117 (2001).

64. Tang, Y. et al. First-principles investigation on redox properties of M -doped CeO 2 (M=Mn, $\mathrm{Pr}, \mathrm{Sn}, \mathrm{Zr}$ ). Phys. Rev. 
B - Condens. Matter Mater. Phys. 82, 1-9 (2010).

65. Chiodelli, G., Flor, G. \& Scagliotti, M. Electrical properties of the ZrO2-CeO2 system. Solid State Ionics 91, 109121 (1996).

66. Fitria, Kanjanaboonmalert, T., Tzu, T. W. \& Sato, K. Electrocatalytic Activity of an SOFC Anode Consisting of Nickel, Cerium Oxide, and Titanium Oxide for the Oxidation of Methane. ECS Trans. 16, 23-29 (2009).

67. Andersson, D. A., Simak, S. I., Skorodumova, N. V., Abrikosov, I. A. \& Johansson, B. Theoretical study of CeO2 doped with tetravalent ions. Phys. Rev. B 76, 174119 (2007).

68. Lane, J. A., Neff, J. L. \& Christie, G. M. Mitigation of the deleterious effect of silicon species on the conductivity of ceria electrolytes. Solid State Ionics 177, 1911-1915 (2006).

69. Fronzi, M., Piccinin, S., Delley, B., Traversa, E. \& Stampfl, C. Water adsorption on the stoichiometric and reduced CeO2(111) surface: A first-principles investigation. Phys. Chem. Chem. Phys. 11, 9188-9199 (2009).

70. Jagdheesh, R., Diaz, M., Marimuthu, S. \& Ocana, J. L. Robust fabrication of $\mu$-patterns with tunable and durable wetting properties: hydrophilic to ultrahydrophobic via a vacuum process. J. Mater. Chem. A 5, 7125-7136 (2017).

71. Li, J., Li, C., Yang, G. \& Li, C. Wettability transition on micro-nano hierarchical structured Ni20Cr coating surface by selective spontaneous adsorption during vacuum evacuation. Mater. Chem. Phys. 219, 292-302 (2018). 